\title{
NOUVELLE
}

\section{Le cil primaire au cour de la pathogénie du prolapsus de la valve mitrale}

Michel Pucéat

> Les malformations congénitales des valves du cœur ont une prévalence estimée à environ $5 \%$ des nouveau-nés, et sont présentes dans $20 \%$ à $30 \%$ des cas de malformation cardio-vasculaire congénitale [1]. La valve mitrale est souvent affectée. Le prolapsus de la valve mitrale plus modéré et sans symptôme est par ailleurs fréquent dans la population générale, affectant 1 individu sur 40, tous âges confondus [2]. Le prolapsus de la valve mitrale congénital non syndromique résulte d'une anomalie du développement embryonnaire de cette valve. Ses conséquences sur la fonction cardiaque ne s'observent cependant, pour la plupart des sujets atteints, qu'à l'âge adulte. Des études génétiques ont identifié, chez les individus atteints, des mutations dans les gènes codant la filamine A (FLNA) [3], la LIM and cysteine-rich domains 1 (LMCDI), la tensine 1 (TNSI) [4], Dachsous (DCHSI) [5], et la zinc-finger protein (DZIPI) [6], mais ces mutations ne rendent compte qu'à peine de $2 \%$ des cas et leurs effets sur le développement de la valve mitrale restent à déterminer. La malformation conduisant au prolapsus de la valve mitrale résulte d'une sécrétion excessive de protéines de la matrice extracellulaire par les cellules interstitielles dans les feuillets valvulaires, ce qui entraîne une dégénérescence myxomateuse ou fibro-élastique de la valve. La fuite de sang vers l'oreillette gauche lors de la contraction ventriculaire (régurgitation mitrale) [7] altère progressivement la fonction du ventricule gauche, nécessitant alors une intervention chirurgicale pour réparer ou changer la valve.

Nous nous sommes intéressés au lien intrigant entre la mutation du gène DCHS1, qui code Dachsous, une protéine impliquée dans la polarité planaire des épithéliums, et le prolapsus de la valve mitrale. En effet, dans le cadre du réseau transatlantique d'excellence MITRAL financé par la Fondation Leducq, nos collaborateurs français et américains avaient identifié, en France et aux États-Unis, deux familles comportant plusieurs individus porteurs, à l'état hétérozygote, d'une mutation faux-sens dans le gène DCHSI (c.6988C >T ; p.Arg2330Cys ou c.7538G>A;
Inserm U1251, université Aix-Marseille, MMG, 27 boulevard Jean Moulin, 13885 Marseille, France.

michel.puceat@inserm.fr

p.Arg2513His) et atteints d'un prolapsus de la valve mitrale [5].

Différenciation de cellules souches pluripotentes induites en cellules valvulaires

Nous avons produit des cellules souches pluripotentes ou cellules iPS (induced pluripotent stem cells) à partir de cellules valvulaires d'un patient porteur de la mutation DCHS1:c.6988C>T prélevées lors d'une intervention chirurgicale sur sa valve mitrale. Des cellules iPS non mutées et les cellules iPS porteuses de la mutation ont été différenciées en cellules endocardiques, puis en cellules valvulaires, par un protocole mimant le scénario du développement normal des valves [8] pendant l'embryogenèse [9] (Figure 1). Les cellules iPS non mutées ont d'abord été différenciées en cellules du mésoderme par l'action de la protéine BMP2 (bone morphogenetic protein 2) et de la protéine Wnt3a (Wingless integration site $3 a$ ), puis en cellules endocardiques par l'action du VEGF (vascular endothelial growth factor) et du FGF8 (fibroblast 


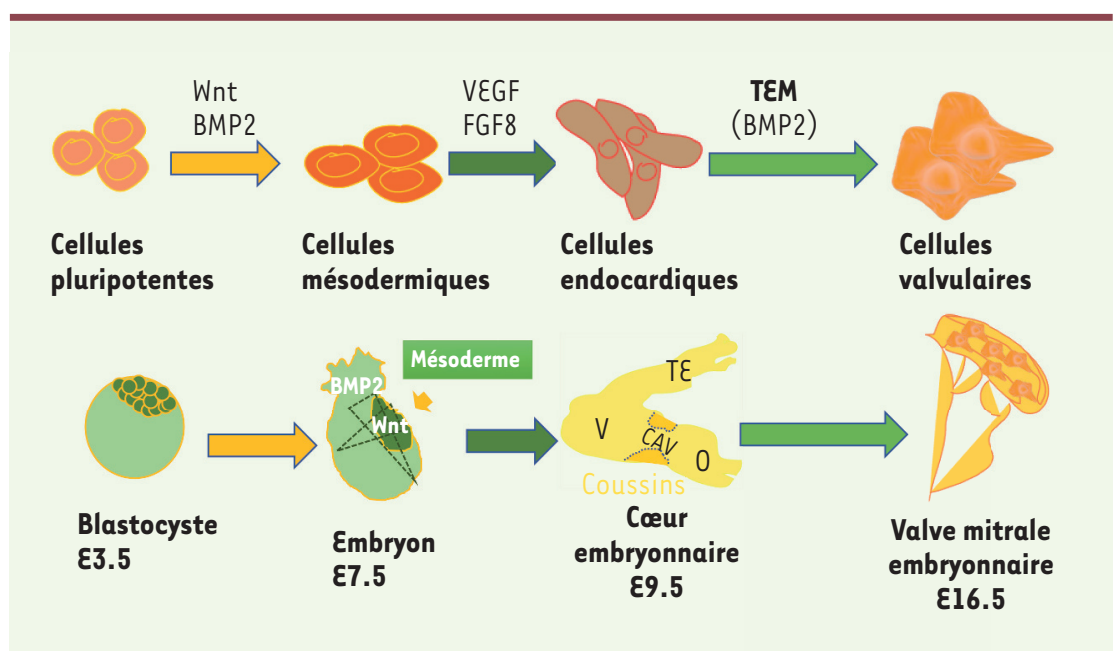

Figure 1. La différenciation de cellules pluripotentes humaines in vitro mime la valvulogenèse embryonnaire chez la souris. TEM : transition épithélio-mésenchymateuse; V : ventricule primaire ; 0 : oreillette ; CAV : canal atrio-ventriculaire ; $T \varepsilon$ : tronc efférent.

growth factor 8). Afin de caractériser le phénotype de ces cellules, nous avons comparé leur transcriptome et celui des cellules endocardiques du canal atrio-ventriculaire de souris au stade embryonnaire 89.5 , au moment de la transition épithélio-mésenchymateuse d'une fraction des cellules endocardiques qui est à l'origine des coussins puis des feuillets valvulaires de la valve mitrale [8]. Cette comparaison a révélé de nombreux transcrits communs aux deux préparations cellulaires. Nous avons confirmé ce résultat en déterminant la séquence nucléotidique des ARN messagers issus de cellules uniques. Cette analyse a révélé l'hétérogénéité des cellules endocardiques obtenues par différenciation des cellules iPS, incluant des cellules hémogéniques ${ }^{1}$ (une possibilité connue de différenciation des cellules endocardiques [10]), mais aussi des cellules endothéliales ou en cours de transition épithélio-mésenchymateuse, c'est-à-dire les cellules à l'origine des valves. Cette analyse a également montré la fiabilité du protocole de différenciation des cellules iPS in vitro (Figure 1). Afin de mimer la situation physiologique, les cellules

${ }^{1}$ Cellule hémogénique : cellule capable de différenciation en cellule hématopoïétique. endocardiques obtenues à partir des cellules iPS non mutées ont ensuite été cultivées en présence de BMP2 (qui est naturellement sécrétée par les cellules du myocarde in vivo) pour déclencher la transition épithélio-mésenchymateuse convertissant ces cellules endocardiques en cellules valvulaires interstitielles. Le séquençage des ARN messagers issus de cellules uniques a montré la diversité des cellules valvulaires interstitielles obtenues, et notamment un début de différenciation vers les types cellulaires attendus dans les différentes couches histologiques de la valve mitrale: fibrosa (riche en protéoglycanes), spongiosa (riche en fibres de collagène), et lamina atrialis ou lamina ventricularis, qui sont les deux couches les plus superficielles situées respectivement du côté atrial et du côté ventriculaire de la valve (riches en fibres d'élastine et de collagène) [11]. L'analyse bioinformatique de ces transcriptomes a montré que les cellules interstitielles se regroupaient en fonction de leur identité cellulaire et de leur localisation future dans la valve. La différenciation des cellules iPS porteuses de la mutation de DCHSI en cellules endocardiques par le même protocole a montré tout d'abord que les cellules obtenues avaient conservé leur capacité de transition épithéliomésenchymateuse en présence de BMP2. Des analyses de protéines de la matrice extracellulaire (collagène, hyaluronane et périostine) par immunofluorescence ont montré une sécrétion accrue de ces protéines par ces cellules. De plus, l'analyse du transcriptome de cellules uniques a montré une perte d'identité des cellules valvulaires interstitielles mutées. Toutes exprimaient tous les gènes codant les protéines de la matrice extracellulaire, et il n'était plus possible de regrouper ces cellules en soustypes correspondant aux différentes couches histologiques. Enfin, l'analyse du cil primaire des cellules interstitielles mutées a révélé un nombre plus faible de cellules ciliées et la présence de cils plus courts, comme cela avait été montré précédemment chez les souris hétérozygotes invalidées pour le gène Dchsl [5].

\section{Le cil primaire au cœur}

de la pathogénie du prolapsus de la valve mitrale

Le cil primaire transmet à la cellule des signaux extérieurs par l'intermédiaire de différents récepteurs membranaires et voies de transduction, dont la voie de Sonic hedgehog (SHH) et son récepteur membranaire Patched 1 [12], connue pour activer l'expression de gènes codant des protéines de la matrice extracellulaire [13]. SHH, par sa liaison à Patched 1 à la surface du cil, induit la translocation de ce récepteur à la surface apicale de la cellule, ce qui déclenche la signalisation intracellulaire. Nous avons fait I'hypothèse que les cellules valvulaires interstitielles porteuses de la mutation de DCHSI dépourvues de cil primaire ou possédant un cil anormalement court étaient incapables de transduire correctement le signal SHH. Une analyse de la localisation de Patched 1 par immunofluorescence a en effet montré que ce récepteur, normalement situé sur le cil primaire des cellules non mutées en l'absence du ligand, était anormalement 


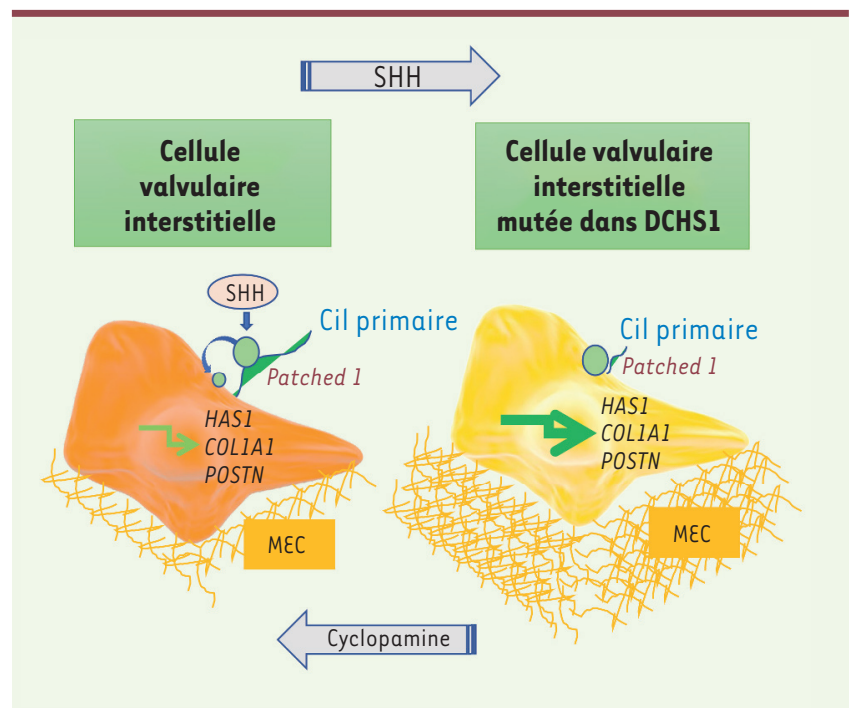

Figure 2. Mécanisme d'activation constitutive de la voie de signalisation de sonic hedgehog (SHH) et de la sécrétion excessive de protéines de la matrice extracellulaire dans les cellules valvulaires mutées. Dans la cellule valvulaire interstitielle normale (à gauche), le récepteur Patched 1, après liaison de son ligand SHH, se déplace du cil primaire vers la surface apicale pour activer la voie de signalisation intracellulaire. SHH active l'expression de gènes codant des protéines de la matrice extracellulaire. Dans la cellule porteuse de la mutation de DCHSI (à droite), Patched 1 est anormalement localisé à la surface apicale même en l'absence de SHH, et la voie de signalisation est constamment activée. La cyclopamine, qui inhibe la voie de signalisation de SHH, corrige le phénotype cellulaire anormal. Inversement, une suractivation de la voie $\mathrm{SHH}$ dans des cellules non mutées leur confère un phénotype pathologique. MEC: matrice extracellulaire; $H A S 1$ : gène codant la hyaluronane synthase 1 ; COLIAl : gène codant la chaîne alpha- 1 du collagène 1 ; POSTN : gène codant la périostine.

présent à la surface apicale des cellules porteuses de la mutation, où son activation constitutive devait stimuler la production de protéines de la matrice extracellulaire. Nous avons alors testé l'efficacité d'un inhibiteur pharmacologique de la voie de $\mathrm{SHH}$, la cyclopamine, sur des cellules mutées, ce qui a permis de rétablir un phénotype cellulaire normal. Inversement, une activation de la voie SHH dans les cellules non mutées par l'ajout d'une forte dose du ligand entraîne un phénotype cellulaire anormal (Figure 2).

\section{Perspectives}

La voie de signalisation par SHH peut être manipulée pharmacologiquement. Elle joue un rôle majeur au cours du développement embryonnaire, et est moins active dans l'organisme adulte (en dehors de certaines maladies telles que le cancer ou la fibrose tissulaire). Une inhibition de la voie SHH pourrait constituer une nouvelle opportunité thérapeutique pour le prolapsus de la valve mitrale chez les individus porteurs de mutations des gènes DCHSI ou DZIPI [6]. On ignore encore si cette voie de signalisation est impliquée dans d'autres formes de prolapsus valvulaire. $\diamond$ The primary cilia at the heart of mitral valve prolapse pathogeny

\section{LIENS D'INTÉRÊT}

L'auteur déclare n'avoir aucun lien d'intérêt concernant les données publiées dans cet article.

\section{RéFÉRENCES}

1. Combs MD, Yutzey KE. Heart valve development: regulatory networks in development and disease. Circ Res 2009 ; 105 : 408-21.

2. Levine RA, Hagège $A A$, Judge $D P$, et al. Mitral valve disease : morphology and mechanisms. Nat Rev Cardiol $2015 ; 12: 689-710$.

3. Kyndt F, Gueffet JP, Probst V, et al. Mutations in the gene encoding filamin $A$ as a cause for familial cardiac valvular dystrophy. Circulation 2007 ; 115 : 40-9.

4. Dina C, Bouatia-Naji N, Tucker N, et al. Genetic association analyses highlight biological pathways underlying mitral valve prolapse. Nat Genet 2015 ; 47 : 1206-11.
5. Durst R, Sauls K, Peal DS, et al. Mutations in DCHSI cause mitral valve prolapse. Nature 2015 ; 525 : 109 13.

6. Toomer KA, Yu M, Fulmer D, et al. Primary cilia defects causing mitral valve prolapse. Sci Transl Med 2019; $11: 493$

7. Delling FN, Vasan RS. Epidemiology and pathophysiology of mitral valve prolapse: new insights into disease progression, genetics, and molecular basis. Circulation $2014 ; 129: 2158-70$.

8. Puceat M. Embryological origin of the endocardium and derived valve progenitor cells: from developmental biology to stem cell-based valve repair. Biochim Biophys Acta 2013 ; 1833 : 917-22.

9. Neri T, Hiriart $\varepsilon$, van Vliet PP, et al. Human prevalvular endocardial cells derived from pluripotent stem cells recapitulate cardiac pathophysiological valvulogenesis. Nat Commun 2019; $10: 1929$.

10. Van Handel B, Montel-Hagen A, Sasidharan R, et al. Scl represses cardiomyogenesis in prospective hemogenic endothelium and endocardium. Cell 2012 ; $150: 590-605$.

11. Schlotter F, Halu A, Goto S, et al. Spatiotemporal multi-omics mapping generates a molecular atlas of the aortic valve and reveals networks driving disease. Circulation $2018 ; 138: 377-93$

12. Rohatgi R, Milenkovic L, Scott MP. Patchedl regulates hedgehog signaling at the primary cilium. Science 2007 ; 317 : 372-6.

13. Liu J, Li Q, Kuehn MR, et al. Sonic hedgehog signaling directly targets hyaluronic acid synthase 2 , an essential regulator of phalangeal joint patterning. Dev Biol 2013 ; 375 : 160-71.

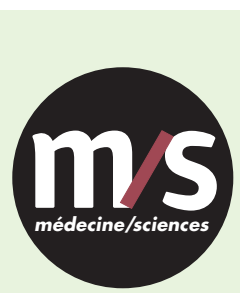

\section{Tarifs d'abonnement $m / s-2019$ \\ Abonnez-vous \\ à médecine/sciences}

$>$ Grâce à $m / s$, vivez en direct les progrès des sciences biologiques et médicales

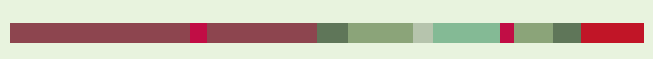

Bulletin d'abonnement page 910 dans ce numéro de $\mathrm{m} / \mathrm{s}$

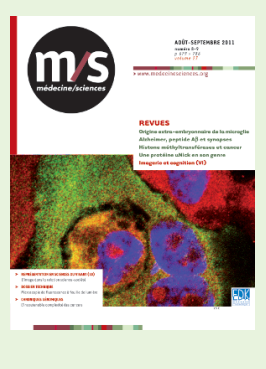

